Unfallchirurg 2009 • 112:364

DOI 10.1007/s00113-009-1633-y

(c) Springer Medizin Verlag 2009

\author{
C. Krettek \\ Unfallchirurgische Klinik, Medizinische Hochschule Hannover
}

\title{
Medizinische Aspekte des Krafttrainings
}

methacin auf die Satellitenzellaktivierung. Es bleibt abzuwarten, in wieweit auch andere nichtsteroidale Antiphlogistika den Effekt von Muskeltraining blockieren und hier Alternativstrategien entwickelt werden können.

M. Jagodzinski und S. Ostermeier zeigen in ihren Beiträgen, dass Krafttraining Schäden sowohl am Gelenkknorpel als auch an den Sehnen und Muskelansätzen hinterlassen kann. Für das patellofemorale Gelenk hat sich ein Wandel in der Ursachenforschung weg von den rein morphologisch-deskriptiven Ursachen wie der Patellaform nach der Wiberg-Klassifikation über das Malalignment hin zu einer multifaktoriellen Entstehung von vorderem Knieschmerz durch Hyperinnervation einiger Schlüsselgewebe (wie z. B. dem lateralen Retinakulum) vollzogen. Gelenkknorpelschäden sind unverändert schwierig zu therapieren und bedürfen besonderer Diagnostik, um die Ursache des Knorpelschadens wie Fehlstellung oder Überbelastung auszuschalten. In wieweit die regenerative Medizin hier in Zukunft einen Beitrag leisten kann, um verlorene Knorpelmasse wieder aufzubauen und die Integrität der Knorpelzellmatrix wieder herzustellen, bleibt abzuwarten.

Unfallchirurgen und Orthopäden werden in Klinik und Praxis häufig mit chronischen rezidivierenden, belastungsabhängigen Beinschmerzen beim sportlich aktiven Menschen konfrontiert, und zwar nicht nur beim Leistungssportler, sondern auch beim Gelegenheitssportler. Wenn differenzialdiagnostisch Stressfrakturen, Myogelosen, ein mediales Tibiastresssyndrom und Neuropathien ausgeschlossen sind, muss v. a. das chronische rezidivierende, belastungsabhängige Kompartmentsyndrom (CRKS) in Betracht gezogen werden. Der Übersichtsartikel von $\mathrm{H}$. Stiegler beleuchtet diese wenig bekannte Entität, die neben den arthrogenen, neurogenen oder muskeltraumatischen Erkrankungen in der besonderen Risikogruppe Sportler eine zunehmende Bedeutung gewinnt.

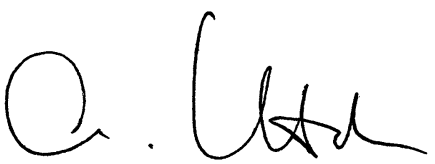

C. Krettek

\section{Korrespondenzadresse}

Prof. Dr. C. Krettek

Unfallchirurgische Klinik,

Medizinische Hochschule Hannover

Carl-Neuberg-Straße 1, 30625 Hannover

krettek.christian@mh-hannover.de 